\title{
Generalistische Ausbildung: Ein Jahr "neue Pflege"
}

\author{
Erste Erfahrungen aus der Umsetzungspraxis Nach jahrzehntelangem Ringen \\ kann seit Anfang diesen Jahres in der Pflege nun generalistisch ausgebildet werden. \\ Wie waren die ersten Monate? Wurden die Erwartungen erfüllt, Chancen genutzt? \\ Ein erster Rückblick auf die - von der Pandemie ebenfalls nicht verschont \\ gebliebene - neue Ausbildung in Schule und Praxis. \& Anke Jakobs, Christine Vogler
}

\section{ZUSAMMENFASSUNG}

Das Pflegeberufegesetz (PflBG) trat am 1. Januar 2020 in Kraft. Mit der ersten Welle der Corona-Pandemie starteten die ersten Jahrgänge bereits im März. Kein leichter Beginn für die neue Pflegeausbildung, die als Hoffnungsträgerin eines ganzen Berufsstandes herhalten muss. Die Autorinnen wagen jetzt den ersten Rückblick auf den Start der Ausbildung. Dabei nehmen sie sowohl die praktische als auch die schulische Ausbildung in den Blick und skizzieren die ersten Eindrücke und Erfahrungen im Kontext des Gesundheitssystems.

\section{Schlüsselwörter: Kostenfaktor, Finanzierung, DRG, PflBG, Pflegeberufegesetz}

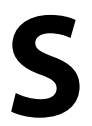

eit Jahrzehnten ringt Deutschland um eine im Bildungssystem durchlässige, international anerkannte und generalistische Ausbildung in den Pflegeberufen(WHO 1986). Nach ca. 40 Jahren Diskussionen, Auswertungen von Modelausbildungsund Studiengängen und politischer Debatten ist 2017 das Pflegeberufereformgesetz verabschiedet worden, und seit 2020 kann in Deutschland Pflege generalistisch ausgebildet werden - an Hochschulen und beruflichen Schulen. Für eine umfangreiche und valide Einschätzung sind wir sicherlich ein wenig früh und das können andere dann besser. Aber erste Eindrücke und Erfahrungen, Chancen und Grenzen zeichnen sich ab. Und genau diese wollen wir hier skizzieren. Dabei werden wir nicht in die Details curricularer Arbeit oder praktischer Ausbildungsportfolios gehen; wir werden das Pflegeberufegesetz aus der Sicht zweier langjähriger verantwortlicher Umsetzerinnen von Pflegeausbildung und Berufsgruppenzugehöriger im System der Gesundheitsversorgung betrachten. Denn das PflBG isoliert anzuschauen, würde der Gesamtsituation nicht gerecht werden.
Eine Vielzahl von Menschen mit ausgewiesener Expertise sorgt momentan mit unglaublich hoher zeitlicher Ressource und großem Kommunikationsaufwand auf allen Ebenen für die Umsetzung des Pflegeberufegesetzes:

- Träger der praktischen Ausbildung (TdpA) entwickeln und setzen - neben der fortlaufenden Patientenversorgung und Ausbildung der Auszubildenden, die vor 2020 gestartet sind - neue Ausbildungspläne um, schließen Kooperationsverträge für die Pflichteinsätze und entwickeln gemeinsam mit den Pflegeschulen neue Ausbildungskonzepte.

_ Pflegeschulen lösen sich - neben dem regulären weitergehenden Schulbetrieb - aus der Kinder-, Kranken- und Altenpflegeperspektive und entwickeln auf Grundlage des bundeseinheitlichen Rahmenlehrplanes kompetenz- und situationsorientierte, an exemplarischen Handlungssituationen ausgerichtete Curricula. Sie schließen Kooperationsverträge mit TdpA und anderen Pflegeschulen, um die notwendigen Voraussetzungen zu erfüllen. Bundesweit beobachten wir zwischen den Schulen Zusammenschlüsse, Fusionen und Anpassungen der Ausbildungskapazitäten.

- Die zuständigen Behörden der Bundesländer schaffen neue Aufsichtsstrukturen im Land und versuchen, im Rahmen der BundLänderkonferenzen, die Verordnungen der Länder soweit es möglich ist, abzustimmen.

- Auf Bundesebene ruft das Gesundheits-, Familien- und Arbeitsministerium im Rahmen der Konzertierten Aktion Pflege (KAP) Bundesländer, Verbände, Gewerkschaften, Sozialkassen und Träger in der Arbeitsgruppe „Ausbildung und Qualifizierung“ zu monatlichen Telefonkonferenzen zusammen, um zu unterstützen und aufkommende Problemfelder zu erkennen. Es wurde eine bundesweite Kampagne („Mach Karriere als Mensch “, BMFSFJ 2019) gestartet, die mit begleitenden Materialien und Fachberatenden Unterstützung bundesweit anbietet (www.pflegeausbildung.net). Das BiBB hat ein Ressort zur Pflegeausbildung aufgebaut und die Fachkommission ist benannt und arbeitet konsequent an der Umsetzung der Neuausrichtung der Curricula. 


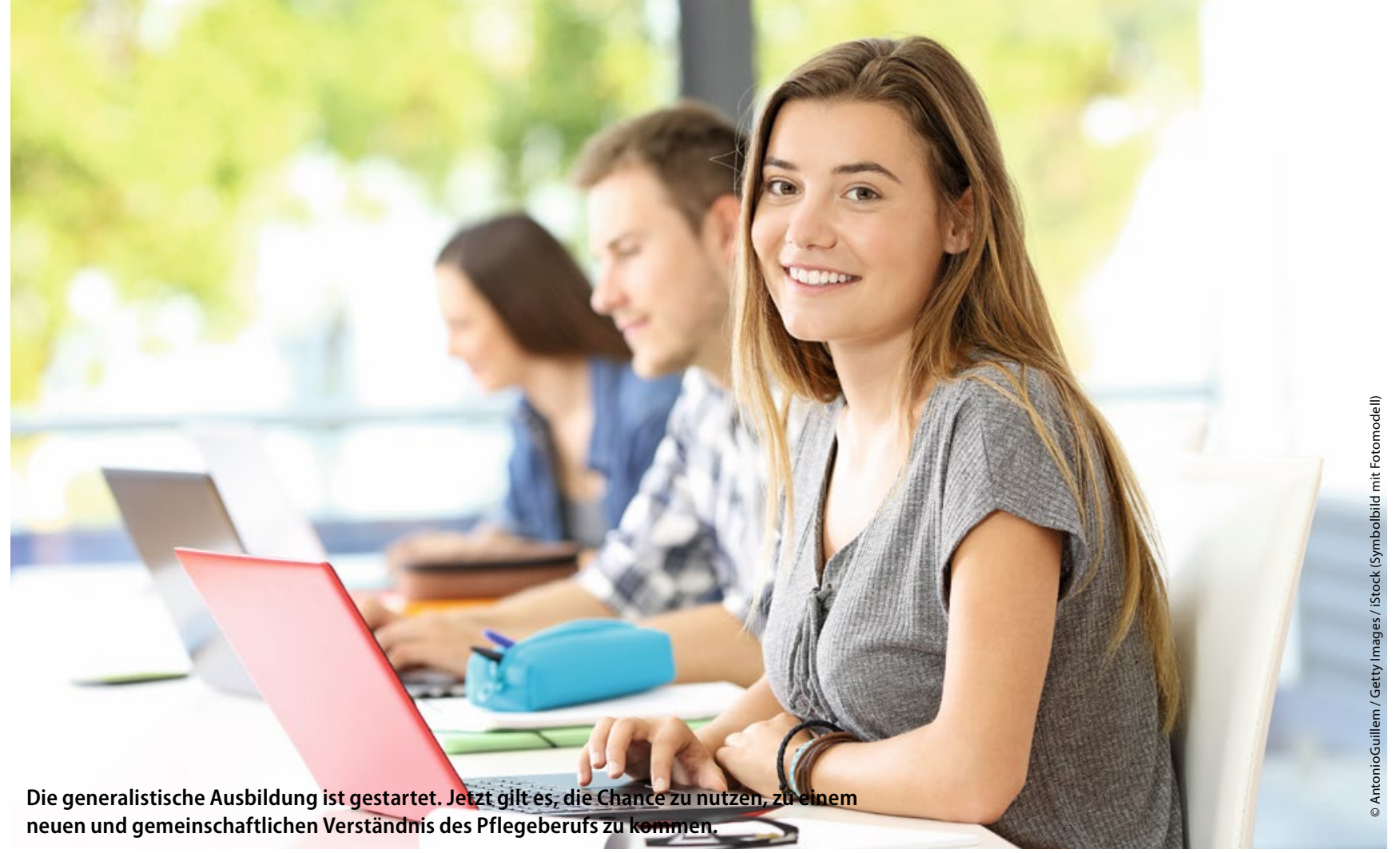

- Verbände und Gewerkschaften, begleiten den Prozess kritisch, um die Rückmeldungen und Vorschläge aus der Umsetzungspraxis von Trägern und Schulen an die Bundes- und Landesebene zu melden.

Die Aufgaben sind hochkomplex. Und vieles ist gut gestartet. Am Ende aber liegt das Gelingen daran, dass die Lehrenden und Praxisanleitenden jede Unterstützung bekommen, die nur denkbar ist. Und genau hier könnte die Gefahrenstelle liegen.

\section{Erfahrungen aus Sicht der Schulen}

Vor allem zwei offene Punkte kristallisieren sich als potenzielle Gefahrenstellen heraus:

Lehrermangel all überall: Es fehlen Lehrende. Und nicht nur ein paar. Neben dem aktuellen Mangel werden in den nächsten Jahren die in den Ruhestand gehenden Babyboomer die nächste Schneise schlagen.

Die seit Jahren ungenügende Ausstattung an staatlichen Studiengängen für die Lehrenden in den Gesundheitsberufen wird in Zukunft dazu führen, dass Schulen Ausbildungskapazitäten nicht bereitstellen können, da es keine Lehrenden auf dem Arbeitsmarkt gibt. Das führt zu solchen Merkwürdigkeiten, dass Bundesländer mal eben per Landesgesetz den im PflBG vorgeschrieben Lehrenden-Lernenden-Schlüssel erhöhen und gleichzeitig die geforderten Qualifikationen der Lehrenden mit zehnjährigen Übergangsfristen absenken (DVO-PflBG NRW).

Die Lehrenden an den Schulen müssten sich zeitlich auf die Umstellung vorbereiten können. Der Schlüssel von 1:20 (Lehrende zu Lernenden) ist in vielen Bundesländern eine Verschlechterung des Bestehenden. Da ist der Hinweis auf den auskömmlichen Ausbildungsfonds irreführend. Unter dem herkömmlichen Schulbetrieb zu erwarten, dass ein neues Berufsverständnis unter den Lehrenden diskutiert und im Rahmen von Curricula umgesetzt werden kann, ist eine schiere Zumutung. An dieser Stelle unsere Anerkennung an die Lehrenden - denn Sie stemmen momentan genau diese Situation. Was es braucht?
— Berufsbegleitende staatliche Studienplätze im Bereich der Pädagogik- und Gesundheitswissenschaften sowohl im Bachelorals auch im Mastersegment. Das sichert familienfreundliche und finanzierbare Studienplätze. Durch Erhöhung und Angebot von Studienplätzen wird zudem noch gesellschaftliche Wertigkeit signalisiert.

- Keine dauerhafte Absenkung von Lehrerqualifikationen. Dafür Anerkennung von Lehramtsstudierenden im begonnenen Master als vollwertige Lehrkräfte mit zeitlicher Fristsetzung. Das würde zumindest erste Abhilfen schaffen - setzt aber berufsbegleitende oder Teilzeitstudiengänge voraus.

Mietkosten als Existenzproblem: Das kann der Bund nicht regeln. Die Länder ringen in ihren Finanzierungsverordnungen um Lösung. Die umfängliche Übernahme gelingt in keinem Bundesland. Die Autorinnen könnten hier viele verzweifelte Beispiele anführen, wie versucht wird, Mietkosten zu sichern, sich Träger und Schulen die Verantwortung für die Mietkosten zuschieben, Länder mit den Schultern zucken und Investitionsförderungen als Mietzuschüsse missbraucht werden sollen und müssen. Fehlendes Finanzvolumen in Unternehmensbilanzen gefährdet die Existenz. Das ist auch bei Schulen nicht anders. Gelingt hier nicht schnellstens eine bundesweite Lösung, werden Ausbildungskapazitäten durch Schulschließungen verloren gehen.

\section{Erfahrungen aus Sicht der praktischen Ausbildung}

Das Pflegeberufegesetz strukturiert das Erlernen des Pflegeberufes neu, die Pflegepraxis zeigt sich jedoch verharrend in den gegebenen Strukturen. Der Ausbildungsauftrag ist immer noch dem Versorgungsauftrag der Patienten nachgeordnet. Positiv zu verzeichnen ist, dass die Praxisanleitenden durch das PflBG gesetzlich geregelt werden. Die geforderten 10\% strukturierte Praxisanleitung müssen für jeden Auszubildenden umgesetzt werden. Dies kommt sehr gut bei den Auszubildenden an. Sie fühlen sich als Auszubildende angenommen, erfahren einen hohen Lerneffekt und können ohne Zeitdruck üben, Fragen stellen, sich auf pflegerische Hand- 
lungen einstellen. Die Auszubildenden erleben in ihrer praktischen Ausbildung aber auch, mit welchem Aufwand dies umgesetzt wird und unter welchen Herausforderungen die Pflegeteams stehen. Das Pflegeberufegesetz ist kein „Pflegepersonalgewinnungsgesetz“. Die Pflegeteams haben im aktuellen Pflegefachkraftmangel mit essentiellen Problemen zu kämpfen, da sich die Ausbildung an sich und im Besonderen gemäß der neuen Vorgaben, sehr schwierig gestalten lässt. Vorherrschende Situation in den pflegerischen Berufsfeldern ist, dass es an qualifiziertem Fachpersonal fehlt und es zu wenige aktive Praxisanleitende gibt. Zahlreiche Pflegeteams verzeichnen eine hohe Wechseldynamik oder arbeiten mit Leasingkräften zusammen. Jeder einzelne dieser Punkte ist ungünstig für eine gute und strukturierte Ausbildung. Zusammengenommen stellen sie viele Praxisanleiterinnen vor fast unlösbare Aufgaben.

Eine große Herausforderung für die Auszubildenden und Pflegenden sind die neue Ausrichtung der Ausbildung. Die Pflegefachlichkeit im Sinne des Pflegeprozesses im primären Fokus zu haben, ist beispielsweise in dem medizinisch orientierten System der Kliniken für alle Akteure neu. So berichten Auszubildende nach ihren ersten Einsätzen in den Kliniken, dass sie oft nach Krankheitsbildern mit den dazugehörigen Krankheitssymptomen gefragt werden und die Durchführung von medizinisch indizierten Maßnahmen im Fokus stehen (z.B. Vitalzeichenkontrolle, Umgang mit intravenösen Zugängen). Prophylaxen, eigene Berufsidentität und $\mathrm{Pa}$ tientenbeobachtung wird dagegen „als normal“ und als „nichts so Besonderes" weitergegeben. Auszubildende bekommen bereits in den ersten Praxiseinsätzen implizit mit, was von den Pflegenden mit hoher Priorität und mit nachgeschalteter Priorität gewertet wird. Dies ist jedoch häufig entgegengesetzt zum neuen Berufsverständnis. Die „neue“ Herangehensweise die Auszubildenden in den ersten Einsätzen als Beobachtende an die Pflege von Pflegebedürftigen heranzuführen, ist für die Pflegeteams unter den aktuellen Personalengpässen schwer nachvollziehbar und in der täglichen Praxis auch nicht vollumfänglich umsetzbar. Hier gilt es im Sinne der Auszubildendenbindung die Kommunikation zwischen den Pflegeteams und den Auszubildenden zu halten, Möglichkeiten und Grenzen zeitnah zu evaluieren, zu reflektieren. Dabei die Personalsituation nicht außen vor zu lassen und gleichzeitig die Umsetzung der generalistischen Ausbildung lebendig und akzeptierend gestalten, ist für die Praxisanleitenden eine große Herausforderung.

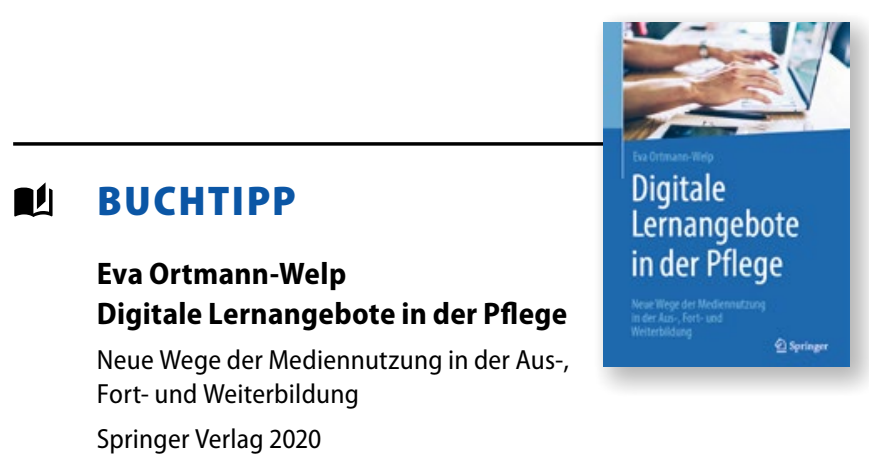

ISBN 978-3-662-61673-4, 35,64€ (Softcover)

ISBN 978-3-662-61674-1, 26,99€ (eBook)

\section{Die Realität bestimmt den „heimlichen Lehrplan“}

Die Pflegepraxis wird von den Auszubildenden mit allen Sinnen und Emotionen wahrgenommen. Das ist eine große Chance - und zugleich ein großes Risiko für das neue gemeinsame Berufsverständnis. Begründete Pflegehandlungen, standardisierte Prozesse und die individuelle Kommunikation als hohen Wert zu sehen und wahrzunehmen setzt voraus, dass die Pflegekräfte dies auch (vor-) leben. Die angespannten Rahmenbedingungen sind auf allen Ebenen zu verändern, und es ist gleichfalls wichtig und notwendig, den Berufsangehörigen der Pflege - und hier vor allem auch den Praxisanleitenden - zu signalisieren, dass sich die Bedingungen in naher Zukunft nachhaltig verändern werden. Praxisanleitende sind die Berufsbildner und setzen die Grundlagen für das Pflegeverständnis - hierfür benötigen sie Zeit und den entsprechenden Freiraum für die Vorbereitung und Durchführung der Übungssequenzen, für Reflexionsgespräche und für individuelle Anleitungssequenzen.

Ausbildung ist zudem ein wichtiges Marketingtool - hier lohnt jede Investition. Sehr positiv werden von den Praxisanleitenden die neu entwickelten Arbeits- und Lernaufgaben angenommen. Diese nehmen die Logik der Praxis auf und sind auf der Grundlage des Rahmenlehrplans für die praktische Ausbildung verfasst. Dies macht den Ausbildungsauftrag neben dem Versorgungsauftrag sichtbar, und der Wert der Ausbildung wird transparenter.

\section{Pflichteinsätze als Problemzonen}

Nach sechs Monaten Ausbildung bei den jeweiligen Ausbildungsträgern wechseln die Auszubildenden im Herbst in die ersten Pflichteinsätze außerhalb des eigenen Ausbildungsträgers. Hier zeichnen sich regional verschiedene Engpässe ab. Das gilt beispielsweise für die ambulanten und pädiatrischen Einsätze. Es bleiben noch viele offene Fragen: Was geschieht, wenn ich als TdpA Pflichteinsätze nicht umfassend organisiert bekomme? Einige Praktikumsorte fordern einen finanziellen Ausgleich. Hier entstehen Ansprüche, die nicht eindeutig gegenfinanziert sind. Wer finanziert beispielsweise die eventuell anfallende Umsatzsteuer? Erschwerend kommt hinzu, dass Kooperationsverhandlungen oft von Konkurrenzdenken geprägt sind und nicht von einem verbindenden Kooperationsgedanken. Der Fachkräftemangel und die ungleichen Gehälter in den verschiedenen Pflegesettings behindern zudem die gemeinsame Ausbildungsidee und die Ausbildungsverpflichtung gegenüber der Gesellschaft.

Für die Auszubildenden sind die zweimal zehn Wochen in einem anderen Pflegesetting nicht nachvollziehbar und stoßen auf wenig Akzeptanz. Sie sehen in der Regel die weiteren Fahrwege, den nicht favorisierten Pflegebereich und das fremde Team als negativen Aspekt. Der Professionsgedanke sowie die Idee, die Pflege in ihren Facetten zu erfahren, ist nicht direkt sichtbar für sie. In der Planung sehen wir zudem eine Überforderung der pädiatrischen und psychiatrischen Bereiche. Hier liegt uns noch kein Erfahrungsbericht vor, jedoch sehen die Praktiker dort die 120-Stunden-Einsätze für jegliche Impulssetzung und die Weitergabe der Fachexpertise als deutlich zu kurz. Hier gilt es, entsprechende Arbeitsaufgaben gemeinsam mit den Teams zu verfassen, damit diese 120-Stunden-Einsätze als Chance und nicht als Belastung gesehen werden. 


\section{Warum der systemische Blick wichtig ist}

Die Grenzen der Umsetzbarkeiten des Gesetzes liegen nicht in dem Gesetz selbst begründet. Für die Pflegeprofession wird es ein großer Schritt nach vorne sein - vor allem die Vorbehaltstätigkeiten und das entstehende gemeinsame Grundverständnis zur eigenen Pflegeprofession und der Blick auf die pflegerischen Kompetenzen, die sich jetzt immer klarer als selbstständiges Handlungsfeld zur Medizin darstellen, generieren hier eine große Chance. Pflege verfolgt immer dieselben Grundsätze: Gesundheit fördern, Krankheit verhüten, Gesundheit wiederherstellen, Leiden lindern. Der Bedarf der Pflege ist universell (ICN/DBfK 2014). Dieser Blick auf die Pflege ist in Deutschland tatsächlich ein Weg, der erst erkannt und dann auch konsequent gegangen werden muss. Die entstandene Spaltung der pflegerischen Profession in Deutschland durch die historisch gewachsenen Ausbildungsstrukturen in Alten-, Kinderund Krankenpflege und durch die systemische Teilung der gesetzlichen Pflege- und Krankenversicherung, entspricht weder dem Anspruch des Berufs noch der Versorgungslage der Pflegebedürftigen und ihrer Angehörigen.

Die Diskussionen um das Pflegeberufegesetz hinterlassen bisweilen den Eindruck, dass es all das heilen soll, was das „System Gesundheit" an Schwierigkeiten produziert hat (Schröder 2009) das wird nicht gelingen. Die unzureichende strukturelle Einbindung der Profession Pflege in das Gesundheitssystem und die unterschiedliche und mangelhafte Finanzierung von pflegerischer Arbeit kann und wird das Pflegeberufegesetz nicht lösen können.

Die Umsetzung des Pflegeberufegesetzes wird sich auch daran messen, was die Absolventen nach der Ausbildung tun können. Was ist, wenn die gut qualifizierten Pflegefachpersonen, die sich nach der Grundausbildung oder dem Studium in das Arbeitsleben stürzen und sich weiterqualifizieren wollen, einem System ausgesetzt sehen, in der die Pflegefachexpertise nichts zählt und Pflege seit Jahrzehnten systematisch abgewertet wurde? In der Pflege ausschließlich Kostenfaktor ist? Und das, was theoretisch gelernt wurde nicht praktisch angewandt werden kann, da medizinische Diagnose, Therapie und Leistungsabrechnung in Deutschland Ärzten vorbehalten ist und damit die Pflegeexpertise im Sinne der „Vorbehaltenen Tätigkeiten" systemisch blockiert wird?

Die formale Umsetzung des Pflegeberufegesetzes wird gelingen. $\mathrm{Ob}$ die politische und gesellschaftliche gelingt? $\mathrm{Da}$ würden die $\mathrm{Au}-$ torinnen jetzt die Glaskugel auspacken müssen. Wir werden jedenfalls weiter mit all den anderen Mitstreitern daran arbeiten!

\section{FAZIT}

Seit Anfang 2020 kann in Deutschland in der Pflege generalistisch ausgebildet werden. Dies birgt die Chance auf ein neues gemeinsames Berufsverständnis der Pflegeberufe.

Die Umsetzung des Pflegeberufegesetzes wird sich unter anderem daran messen, was die Absolventen nach der Ausbildung tun können und wie sie ihre Fachexpertise im Gesundheitssystem einbringen können.

\section{Literatur}

_ BMFSFJ (2019),,Mach Karriere als Mensch“. https://www.pflegeausbildung.net/ausbildungsoffensive-und-kampagne.html

_ BMFSFJ (2019) https://www.pflegeausbildung.net

_ BMG, BMFSFJ, BMAS (2019) Konzertierte Aktion Pflege. Berlin https://www.bundesgesundheitsministerium.de/konzertierte-aktion-pflege.html

_ Bohrer A, Walter A (2015) Entwicklung beruflicher Identität - empirische Erkenntnisse zum Lernen in der Berufspraxis. Pädagogik der Gesundheitsberufe 2 (3) 23-31

_ Bohrer A (2013) In der Praxis lernen. Padua 8 (2) 85-93

_ Bohrer A (2013) Selbstständig werden in der Pflegepraxis. Wissenschaftlicher Verlag, Berlin

_ Fichtmüller F, Walter A (2007) Pflegen lernen. V\&R unipress, Göttingen

_ ICN Ethikcodex - Text aus der Präambel, Übersetzung DBfK 2014

_ Neuweg GH (2004) Könnerschaft und implizites Wissen. Waxmann, Münster

_ Schröder T (2009) Arbeits- und Lernaufgaben für die arbeitsprozessintegrierte beruflich-betriebliche Weiterbildung - Ergebnisse aus einem Handlungsforschungsprojekt. In: bwp@Berufs-und Wirtschaftspädagogik - online. 17, 1-23

_ Verordnung zur Durchführung des Pflegeberufegesetzes in Nordrhein-Westfalen (Durchführungsverordnung PflegeberufegesetzDVO-PflBG NRW)

_ WHO (1986) Gesundheit für alle bis zum Jahr 2000. https://www. euro.who.int/_data/assets/pdf_file/0006/129534/Ottawa_ Charter_G.pdf (WHO-autorisierte Übersetzung: Hildebrandt) Kickbusch)

_ WHO (1999) Gesundheit 21 - Gesundheit für alle im 21. Jahrhundert. WHO, Europäische Schriftenreihe, Nr. 6

_ WHO (1999) Pflege und Hebammenwesen in Europa, München. Übersetzung DBFK, Eschborn

\section{Autorenkontakt}

Anke Jakobs, Kinderkrankenschwester, Dipl. Pflegepädagogin, Tutor der Pflegewissenschaft, M.A. Schulleitungsmanagement, 10 Berufserfahrung in der Kinderkrankenpflege, 13 Jahre Berufserfahrung in der Pflegebildung, heute Leiterin der praktischen Ausbildung Vivantes Netzwerk für Gesundheit $\mathrm{GmbH}$

E-Mail: Anke.Jakobs@vivantes.de

Christine Vogler, Krankenschwester, Diplompflegepädagogin, 30 Jahre Gesundheitswesen, 15 Jahre Schulleitung an den Wannseeschulen und seit 2004 mit Umsetzung und Etablierung einer generalistischen Ausbildung vertraut, heute Geschäftsführerin Berliner Bildungscampus für Gesundheitsberufe gGmbH.

E-Mail: christine.vogler@bildungscampus-berlin.de 